\title{
SPECTRUM ASYMPTOTICS FOR ONE "NONSMOOTH" VARIATIONAL PROBLEM WITH SOLVABLE CONSTRAINT
}

\author{
A. B. ALEKSEEV, M. SH. BIRMAN, AND N. D. FILONOV
}

\begin{abstract}
In a previous paper by Birman and Filonov, the spectrum of the Maxwell operator with nonsmooth coefficients in Lipschitz domains was investigated. The claim that its eigenvalues obey the Weyl asymptotics was proved up to a statement about the spectrum of an auxiliary problem with constraint. The proof of that statement is given in the present paper.
\end{abstract}

\section{§1. INTRODUCTION}

In the paper [9], the Weyl spectrum asymptotics was established for the electromagnetic oscillations of a resonator with perfectly conductive boundary. Such an asymptotics for an empty resonator with smooth boundary was found, for the first time, by H. Weyl [11] in 1912. The presence of a medium in the domain under consideration, lifting the smoothness requirements on the coefficients that characterize the properties of this medium (in the vacuum case these coefficients are constant), and the nonsmoothness of the boundary of the domain require overcoming hard mathematical difficulties. The condition on the resonator boundary that was found in [9] is half-effective (see Condition 5.1 in [9]); the bounded domains with Lipschitz boundary, as well as the domains with screens described in [8] satisfy this condition.

In [9], the proof of the Weyl asymptotic formula was reduced to a proposition about the spectral asymptotics of some variational problem (see Theorem 6.1 in [9] or Theorem 3.2 below). The present paper is devoted to the proof of that proposition, thus completing the investigation [9] (we wished that [9] be free of technical considerations).

Note that the Weyl type asymptotics for arbitrary measurable coefficients but for smooth boundary of the resonator $\Omega$ was justified in 1976. In [2], the corresponding result was obtained as a consequence of more general facts. Unfortunately, no full exposition of the results of the short note [2] has ever been published. However, the full proofs were given in the thesis [1]. On the other hand, the Weyl formula for an empty resonator $\Omega$ with Lipschitz boundary was justified in [6]. It turned out that combining the methods of $[1,2,6]$ is not at all automatic. The corresponding analysis was done in [9], where the question was reduced to the spectral asymptotics of one problem with constraints under the Dirichlet boundary condition, which is much simpler than the conditions of perfect conductivity. The main Theorem 3.2 of the present paper contains the necessary result. Note that now the variational problem is posed on the Sobolev space $H_{0}^{1}$, so that the corresponding asymptotic formula is true for all bounded domains.

The proof of Theorem 3.2 can also be extracted from the more general results of $[1,2]$. But here we give a direct proof, which is, of course, simpler. Next, the text [1] is not a formal publication and is hardly available.

2000 Mathematics Subject Classification. Primary 35P20.

Key words and phrases. Weyl spectrum asymptotics, resonator with perfectly conductive boundary, spectrum of the quotient of quadratic forms, problem with constraint. 
The symbols $\langle\cdot, \cdot\rangle$ and $|\cdot|$ denote the scalar product and the norm in $\mathbb{C}^{3} ;(\cdot, \cdot)$ and $\|\cdot\|$ denote the scalar product and the norm in $L_{2}(\Omega, \mathbb{C})$ or in $L_{2}\left(\Omega, \mathbb{C}^{3}\right)$, where $\Omega$ is an open bounded set in $\mathbb{R}^{3}$; and $\|\cdot\|_{L_{p}}$ is the norm in $L_{p}(\Omega)$. We denote by $H^{l}$ the Sobolev space of scalar-valued or vector-valued functions, and by $H_{0}^{1}$ the closure of the set $C_{0}^{\infty}$ in the $H^{1}$-norm. For an open cube $Q$ in $\mathbb{R}^{3}$, we introduce also the spaces $\tilde{H}^{l}(Q)$ and $\tilde{C}^{\infty}(Q)$ of functions in $H^{l}(Q)$ or $C^{\infty}(\bar{Q})$, respectively, that satisfy the periodic boundary conditions. By the spectrum of the quotient of two quadratic forms we mean the following. The form in the denominator determines a metric in a Hilbert space, the form in the numerator determines a selfadjoint operator in this space, and the spectrum of this operator is called the spectrum of the quotient of the quadratic forms in question.

In $\S \S 2$ and 3, we formulate the main result. In $\S 4$, auxiliary facts on compact operators are cited. In $\S \S 5-8$, we consider some asymptotic functionals and prove their continuity with respect to the coefficients of differential operators. In $\S 9$ we study a model periodic problem with constant coefficients. The proof of the main result is completed in $\S 10$.

\section{§2. Setting of the Problem}

Let $\Omega \subset \mathbb{R}^{3}$ be an open bounded set. We regard the form

$$
\int_{\Omega}|\nabla \varphi|^{2} d x, \quad \varphi \in H_{0}^{1}(\Omega, \mathbb{C})
$$

as the basic metric form on the space $H_{0}^{1}(\Omega, \mathbb{C})$, and the form

$$
\int_{\Omega}|\nabla w|^{2} d x=\int_{\Omega}\left(|\operatorname{curl} w|^{2}+|\operatorname{div} w|^{2}\right) d x, \quad w \in H_{0}^{1}\left(\Omega, \mathbb{C}^{3}\right),
$$

as the basic metric form on the space $H_{0}^{1}\left(\Omega, \mathbb{C}^{3}\right)$. Let $b, \mu, f$ be three measurable $(3 \times 3)$-matrix-valued functions such that

$$
0<\underline{b} \mathbb{1} \leq b(x) \leq \bar{b} \mathbb{1}
$$

and

$$
0<\underline{\mu} \mathbb{1} \leq \mu(x) \leq \bar{\mu} \mathbb{1}
$$

almost everywhere, where $\mathbb{1}$ is the unit matrix. Let

$$
f \in L_{3}(\Omega) \text {. }
$$

We put

$$
B[\varphi, \varphi]=\int_{\Omega}\langle b \nabla \varphi, \nabla \varphi\rangle d x, \quad \varphi \in H_{0}^{1}(\Omega, \mathbb{C}) .
$$

By (2.3), the form $B$ is metrically equivalent to the form (2.1).

For $w \in H_{0}^{1}\left(\Omega, \mathbb{C}^{3}\right)$, let $\varphi \in H_{0}^{1}(\Omega, \mathbb{C})$ be a solution of the equation

$$
\operatorname{div}(b \nabla \varphi-f w)=0,
$$

which is understood in the following sense:

$$
(b \nabla \varphi-f w, \nabla \zeta)=0 \quad \text { for all } \zeta \in H_{0}^{1}(\Omega, \mathbb{C}) .
$$

Since the imbedding $H_{0}^{1}(\Omega) \subset L_{6}(\Omega)$ is continuous, equation (2.7) (under condition (2.5)) has a unique solution $\varphi \in H_{0}^{1}(\Omega, \mathbb{C})$. The linear operator

$$
\Psi: w \mapsto \varphi, \quad \Psi: H_{0}^{1}\left(\Omega, \mathbb{C}^{3}\right) \rightarrow H_{0}^{1}(\Omega, \mathbb{C}),
$$

is continuous. Below we shall show that it is compact.

Equation (2.7) implies that

$$
(b \nabla \varphi, \nabla \varphi)=(f w, \nabla \varphi) \leq \underline{b}^{-1 / 2}\|f w\|(b \nabla \varphi, \nabla \varphi)^{1 / 2},
$$


whence

$$
B[\Psi w, \Psi w] \leq \underline{b}^{-1}\|f w\|^{2}, \quad w \in H_{0}^{1}\left(\Omega, \mathbb{C}^{3}\right) .
$$

We introduce the following quadratic form $K$ :

$$
\begin{aligned}
& K[w, w] \equiv K_{b, f}[w, w]=\left(b^{-1} f w, f w\right)-B[\Psi w, \Psi w] \\
& \quad=\left(b^{-1}(f w-b \nabla(\Psi w)), f w-b \nabla(\Psi w)\right), \quad w \in H_{0}^{1}\left(\Omega, \mathbb{C}^{3}\right) .
\end{aligned}
$$

Evidently, the nonnegative form $K$ is continuous on the space $H_{0}^{1}\left(\Omega, \mathbb{C}^{3}\right)$. Below we shall see that this form is compact.

Fixing $\alpha>0$, we introduce the form

$$
A[w, w]=\left(\mu^{-1} \operatorname{curl} w, \operatorname{curl} w\right)+\alpha\|\operatorname{div} w\|^{2}, \quad w \in H_{0}^{1}\left(\Omega, \mathbb{C}^{3}\right) .
$$

By (2.4), this form is equivalent to the form (2.2).

Now we consider the variational quotient

$$
K[w, w] / A[w, w], \quad w \in H_{0}^{1}\left(\Omega, \mathbb{C}^{3}\right) .
$$

The numerator of this quotient contains a nonlocal operator $\Psi: w \mapsto \varphi$. The quotient (2.12) is local with respect to the pair $w, \varphi$, but the arguments $w, \varphi$ satisfy the (solvable) constraint (2.7). In [2], the asymptotics of the spectrum for a wide class of variational problems with constraints was established. Here, our goal is to write down the asymptotics in the case described above (see Theorem 3.2 below), and to present its full proof.

\section{§3. Formulation of the Result}

Let $\Omega \subset \mathbb{R}^{3}$ be an open bounded set, and let $b, \mu, f$ be matrices defined on $\Omega$ and satisfying conditions (2.3)-(2.5). For $x \in \Omega$ and $\xi \in \mathbb{R}^{3} \backslash\{0\}$, we consider the following algebraic spectral problem:

$$
\begin{aligned}
& f(x)^{*} b(x)^{-1} f(x) h-\langle b(x) \xi, \xi\rangle^{-1}\left\langle h, f(x)^{*} \xi\right\rangle f(x)^{*} \xi \\
& \quad=\lambda\left(r(\xi) \mu(x)^{-1} r(\xi) h+\alpha\langle h, \xi\rangle \xi\right), \quad 0 \neq h \in \mathbb{C}^{3},
\end{aligned}
$$

where the matrix $r(\xi)$ is the symbol of the differential operator curl:

$$
r(\xi)=i\left(\begin{array}{ccc}
0 & \xi_{3} & -\xi_{2} \\
-\xi_{3} & 0 & \xi_{1} \\
\xi_{2} & -\xi_{1} & 0
\end{array}\right) .
$$

Let $\lambda_{j}(x, \xi), j=1,2,3$, denote the eigenvalues of (3.1). Problem (3.1) has the following properties.

- For $\xi \neq 0$, the quadratic form of the matrix on the right-hand side is positive definite.

- The quadratic form of the matrix on the left-hand side of (3.1) can be represented as

$$
\begin{aligned}
& \left\langle f^{*} b^{-1} f h, h\right\rangle-\langle b \xi, \xi\rangle^{-1}\left\langle h, f^{*} \xi\right\rangle\left\langle f^{*} \xi, h\right\rangle \\
& \quad=\left\langle b^{-1}\left(f h-\langle b \xi, \xi\rangle^{-1}\left\langle h, f^{*} \xi\right\rangle b \xi\right), f h-\langle b \xi, \xi\rangle^{-1}\left\langle h, f^{*} \xi\right\rangle b \xi\right\rangle
\end{aligned}
$$

therefore, it is nonnegative. Consequently, all the eigenvalues are nonnegative: $\lambda_{j}(x, \xi) \geq 0$.

- If $f(x)=0$, then the left-hand side vanishes identically. Hence,

$$
f(x)=0 \Longrightarrow \lambda_{1}(x, \xi)=\lambda_{2}(x, \xi)=\lambda_{3}(x, \xi)=0 .
$$

- For a fixed $x \in \Omega$, the functions $\lambda_{j}(x, \xi)$ are continuous in $\xi \in \mathbb{R}^{3} \backslash\{0\}$ and homogeneous of order -2 , i.e., $\lambda_{j}(x, t \xi)=t^{-2} \lambda_{j}(x, \xi)$. 
We introduce the counting function for problem (3.1):

$$
n(\lambda ; x, \xi)=\#\left\{j: \lambda_{j}(x, \xi)>\lambda\right\}, \quad \lambda>0 .
$$

Proposition 3.1. Let $x \in \Omega$. We have

$$
\int_{\mathbb{R}^{3}} n(\lambda ; x, \xi) d \xi=\frac{1}{3 \lambda^{3 / 2}} \int_{|\theta|=1} \sum_{j=1}^{3} \lambda_{j}(x, \theta)^{3 / 2} d S(\theta) .
$$

Proof. The homogeneity of $\lambda_{j}$ implies that, for $\xi=\rho \theta$ with $\rho>0$, we have

$$
\lambda_{j}(x, \xi)>\lambda \Longleftrightarrow \lambda_{j}(x, \theta)>\rho^{2} \lambda \text {. }
$$

Therefore,

$$
\begin{aligned}
& \int_{\mathbb{R}^{3}} n(\lambda ; x, \xi) d \xi=\sum_{j=1}^{3} \int_{\left\{\xi: \lambda_{j}(x, \xi)>\lambda\right\}} d \xi \\
& \quad=\sum_{j=1}^{3} \int_{|\theta|=1} \int_{0}^{\left(\lambda_{j}(x, \theta) / \lambda\right)^{1 / 2}} \rho^{2} d \rho d S(\theta)=\frac{1}{3 \lambda^{3 / 2}} \int_{|\theta|=1} \sum_{j=1}^{3} \lambda_{j}(x, \theta)^{3 / 2} d S(\theta) .
\end{aligned}
$$

Now we can formulate the main result of the paper.

Theorem 3.2. Let $\Omega \subset \mathbb{R}^{3}$ be an open bounded set. The spectrum of the quotient (2.12) of the quadratic forms $K$ and $A$ defined in $§ 2$ has the following asymptotics:

$$
n(\lambda,(2.12)) \sim(2 \pi)^{-3} \int_{\Omega} \int_{\mathbb{R}^{3}} n(\lambda ; x, \xi) d x d \xi, \quad \lambda \rightarrow+0 .
$$

Here $n(\lambda,(2.12))$ is the counting function of the spectrum of the quotient $(2.12), n(\lambda ; x, \xi)$ is the counting function of the $\lambda$-spectrum of problem (3.1), and the symbol $\sim$ means that the ratio of the two sides of (3.4) tends to 1 as $\lambda \rightarrow+0$.

Remark 3.3. Routine calculations show that the integral on the right in (3.4) is continuous with respect to variation of the coefficients $f, \mu$, and $b$ in the $L_{3}(\Omega)$-norm.

We also consider a problem with periodic boundary conditions. Let $Q$ be a cube in $\mathbb{R}^{3}$. Recall that $\tilde{C}^{\infty}(Q)$ and $\tilde{H}^{l}(Q)$ denote the spaces of functions in $C^{\infty}(\bar{Q})$ and $H^{l}(Q)$, respectively, that satisfy periodic boundary conditions. Suppose that the matrices $b, \mu$, and $f$ are defined on $Q$ and satisfy (2.3)-(2.5), as above. We introduce the quadratic forms

$$
\begin{aligned}
& \tilde{B}[\varphi, \varphi]=(b \nabla \varphi, \nabla \varphi), \quad \varphi \in \tilde{H}^{1}(Q, \mathbb{C}), \\
& \tilde{A}[w, w]=\left(\mu^{-1} \operatorname{curl} w, \operatorname{curl} w\right)+\alpha\|\operatorname{div} w\|^{2}, \quad w \in \tilde{H}^{1}\left(Q, \mathbb{C}^{3}\right),
\end{aligned}
$$

where $\alpha>0$ is the same as in $(2.11)$. Let $\tilde{\Psi}: \tilde{H}^{1}\left(Q, \mathbb{C}^{3}\right) \rightarrow \tilde{H}^{1}(Q, \mathbb{C})$ be the operator that takes $w$ to the solution $\tilde{\varphi}$ of the equation

$$
(b \nabla \tilde{\varphi}-f w, \nabla \zeta)=0, \quad \zeta \in \tilde{H}^{1}(Q, \mathbb{C}),
$$

satisfying the condition $\int_{Q} \tilde{\varphi} d x=0$. Finally, we define a form $\tilde{K}$ by the formula

$$
\tilde{K}[w, w] \equiv \tilde{K}_{b, f}[w, w]=\left(b^{-1} f w, f w\right)-\tilde{B}[\tilde{\Psi} w, \tilde{\Psi} w], \quad w \in \tilde{H}^{1}\left(Q, \mathbb{C}^{3}\right) .
$$

In order to prove Theorem 3.2, in addition to the variational problem (2.12) with the Dirichlet boundary conditions, we shall also use a similar problem on the spectrum of the variational quotient

$$
\tilde{K}[w, w] / \tilde{A}[w, w], \quad w \in \tilde{H}^{1}\left(Q, \mathbb{C}^{3}\right), \quad \int_{Q} w d x=0
$$


under the periodic boundary conditions. Observe that the last condition in (3.8) eliminates the degeneracy of the form $\tilde{A}$ on constant vectors.

\section{§4. Auxiliary FACTS}

Here we collect necessary results about compact operators. Let $S$ be a compact positive operator on a Hilbert space $\mathcal{H}$; its consecutive eigenvalues are denoted by $\lambda_{k}(S)$. For a compact operator $T$ from a Hilbert space $\mathcal{H}$ to a Hilbert space $\mathcal{N}$, its singular numbers $s_{k}(T)$ are defined by the formula $s_{k}(T)=\lambda_{k}\left(T^{*} T\right)^{1 / 2}$. Let

$$
n(s, T)=\#\left\{k: s_{k}(T)>s\right\}, \quad s>0,
$$

stand for the counting function for the sequence $\left\{s_{k}(T)\right\}$. An equivalent definition of this function can be based on the minimaximal properties of the singular numbers:

$$
n(s, T)=\max \operatorname{dim} L, \quad\|T x\|_{\mathcal{N}}>s\|x\|_{\mathcal{H}} \quad \text { for all } x \in L \backslash\{0\} .
$$

Here $L \subset \mathcal{H}$ is a subspace. In the case where the spaces coincide, $\mathcal{H}=\mathcal{N}$, and $T$ is a positive operator, we have $s_{k}(T)=\lambda_{k}(T)$, and

$$
n(s, T)=\max \operatorname{dim} L, \quad(T x, x)>s\|x\|^{2}, x \in L \backslash\{0\} .
$$

If $T$ is selfadjoint (not necessarily positive) and $2 T_{ \pm}=|T| \pm T$, then

$$
n(s, T)=n\left(s, T_{+}\right)+n\left(s, T_{-}\right) .
$$

Let $q \geq 1$. For a compact operator $T: \mathcal{H} \rightarrow \mathcal{N}$, we introduce the asymptotic functionals

$$
\Delta_{q}(T)=\limsup _{s \rightarrow 0} s^{q} n(s, T), \quad \delta_{q}(T)=\liminf _{s \rightarrow 0} s^{q} n(s, T),
$$

which can have zero, positive, or infinite values. In the sequel, the symbol $d_{q}(T)$ denotes any of the functionals (4.3). We list some properties of these functionals (see, e.g., [5] and $[7])$. We have

$$
d_{q}\left(T^{*}\right)=d_{q}(T)
$$

and

$$
\begin{gathered}
d_{q}\left(T_{1}+T_{2}\right)^{1 /(q+1)} \leq d_{q}\left(T_{1}\right)^{1 /(q+1)}+\Delta_{q}\left(T_{2}\right)^{1 /(q+1)} \\
\left|d_{q}\left(T_{1}\right)^{1 /(q+1)}-d_{q}\left(T_{2}\right)^{1 /(q+1)}\right| \leq \Delta_{q}\left(T_{1}-T_{2}\right)^{1 /(q+1)} .
\end{gathered}
$$

Proposition 4.1. Let $\mathcal{H}, \mathcal{N}_{1}$, and $\mathcal{N}_{2}$ be Hilbert spaces. Let $T_{1}$ be a bounded operator from $\mathcal{N}_{1}$ to $\mathcal{N}_{2}$, and let $T_{2}$ be a compact operator from $\mathcal{H}$ to $\mathcal{N}_{1}$. Then

$$
\Delta_{q}\left(T_{1} T_{2}\right) \leq\left\|T_{1}\right\|^{q} \Delta_{q}\left(T_{2}\right) .
$$

Moreover, if $\Delta_{q}\left(T_{2}\right)<\infty$ and $T_{1}$ is compact, then $\Delta_{q}\left(T_{1} T_{2}\right)=0$.

Also, we have

$$
\Delta_{q}\left(T_{1} T_{2}\right) \leq 2 \Delta_{2 q}\left(T_{1}\right)^{1 / 2} \Delta_{2 q}\left(T_{2}\right)^{1 / 2} .
$$

Proposition 4.2. Let $T, T_{n}$ be compact operators from $\mathcal{H}$ to $\mathcal{N}$. If $\Delta_{q}\left(T-T_{n}\right) \rightarrow 0$ as $n \rightarrow \infty$, then $d_{q}\left(T_{n}\right) \rightarrow d_{q}(T)$. In particular, if $\Delta_{q}\left(T_{0}\right)=0$, then $d_{q}\left(T+T_{0}\right)=d_{q}(T)$.

Proposition 4.3. Let $T, T_{1}$, and $T_{2}$ be selfadjoint operators in a Hilbert space $\mathcal{H}$, and let the operators $T_{1}$ and $T_{2}$ be positive. Suppose that

$$
|(T x, x)| \leq\left(T_{1} x, x\right)^{1 / 2}\left(T_{2} x, x\right)^{1 / 2}, \quad x \in \mathcal{H} .
$$


Then

$$
\Delta_{q}(T) \leq 4 \Delta_{q}\left(T_{1}\right)^{1 / 2} \Delta_{q}\left(T_{2}\right)^{1 / 2} .
$$

If $T>0$, then the factor 4 in (4.10) can be replaced by 2 .

If $T>0$, then Proposition 4.3 can be deduced easily from (4.5); the general case follows by $(4.2)$.

Proposition 4.4. Let $T$ and $S=S^{*}$ be compact operators on $\mathcal{H}$. Suppose that the form $(u, v)_{1}=(u, v)-(S u, v)$ is such that $(u, u)_{1}>0$ for $u \neq 0$. Then $(u, v)_{1}$ determines a new inner product in $\mathcal{H}$, and the new norm is equivalent to the initial one. Let $T_{1}$ be the operator defined by the identity $\left(T_{1} u, v\right)_{1}=(T u, v), u, v \in \mathcal{H}$. Then $d_{q}\left(T_{1}\right)=d_{q}(T)$.

In $[9, \S 5.3]$, we used the statement that the asymptotic limits $d_{q}$ for a quotient of two quadratic forms do not change under perturbation of the form in the denominator by a relatively compact term. Proposition 4.4 is an exact form of that statement.

We also mention the following fact, which is contained in the results of the paper [3].

Proposition 4.5. Let $T_{1}$ and $T_{2}$ be compact nonnegative operators in $\mathcal{H}$. Then

$$
\Delta_{2 q}\left(T_{1}^{1 / 2}-T_{2}^{1 / 2}\right) \leq \Delta_{q}\left(T_{1}-T_{2}\right) .
$$

All propositions of this section will be used below with $q=3 / 2$. When applying them to the spectrum of the quotient of two quadratic forms, we assume that the form in the denominator determines a scalar product in $\mathcal{H}$, and the form in the numerator corresponds to a (compact) operator acting on $\mathcal{H}$.

\section{$\S 5$. Continuity of the functionals $d_{3 / 2}((2.12))$ And $d_{3 / 2}((3.8))$ WITH RESPECT TO THE COEFFICIENT $f$}

For the proof of Theorem 3.2, we need to establish the continuity of the functionals $d_{3 / 2}((2.12))$ and $d_{3 / 2}((3.8))$ with respect to the coefficients. In this section we assume that the matrix-valued functions $\mu$ and $b$ are fixed and we prove that these functionals are continuous in the coefficient $f$. Continuity with respect to $\mu$ and $b$ will be established in $\S 8$.

We consider the quotient $\|f w\|^{2} /\|w\|_{H^{1}}^{2}$ either with the Dirichlet condition, or in the cube $Q$ with the periodic boundary conditions. For the counting function of the spectrum of the quotients

$$
\begin{aligned}
& \|f w\|^{2} /\|w\|_{H^{1}}^{2}, \quad w \in H_{0}^{1}(\Omega), \\
& \|f w\|^{2} /\|w\|_{H^{1}}^{2}, \quad w \in \tilde{H}^{1}(Q),
\end{aligned}
$$

we have the inequalities

$$
\lambda^{3 / 2} n(\lambda,(5.1)) \leq C_{0}\|f\|_{L_{3}}^{3}, \quad \lambda^{3 / 2} n(\lambda,(5.2)) \leq C_{0}\|f\|_{L_{3}}^{3},
$$

where $C_{0}$ is an absolute constant. The first inequality is none other than the famous Rosenblum-Lieb-Cwikel estimate in the 3-dimensional case. The second inequality can be obtained in a similar way (see [10]) by using the Fourier series instead of the Fourier integral. Clearly, (5.3) yields

$$
\Delta_{3 / 2}((5.1)) \leq C_{0}\|f\|_{L_{3}}^{3}, \quad \Delta_{3 / 2}((5.2)) \leq C_{0}\|f\|_{L_{3}}^{3} .
$$

Recalling (2.9), we obtain

$$
\Delta_{3}(\Psi), \Delta_{3}(\tilde{\Psi}) \leq C\|f\|_{L_{3}}^{3 / 2},
$$

where $\Psi$ is understood as an operator from $H_{0}^{1}\left(\Omega, \mathbb{C}^{3}\right)$ to $H_{0}^{1}(\Omega, \mathbb{C})$, and $\tilde{\Psi}$ as an operator from $\tilde{H}^{1}\left(Q, \mathbb{C}^{3}\right)$ to $\tilde{H}^{1}(Q, \mathbb{C})$. 
Now, let $f_{j}, j=1,2$, be two matrices satisfying (2.5), and let $\Psi_{j}$ be the corresponding operator $\Psi$ with $f=f_{j}$. Let $T_{j}$ be the operator corresponding to the form $K_{b, f_{j}}$ in the Hilbert space $H_{0}^{1}\left(\Omega, \mathbb{C}^{3}\right)$ with the metric form (2.11). By (4.6), it suffices to estimate $\Delta_{3 / 2}\left(T_{1}-T_{2}\right)$ in terms of $\left\|f_{1}-f_{2}\right\|_{L_{3}(\Omega)}$. Consider the form

$$
\begin{aligned}
& K_{12}[w, w]:=K_{b, f_{1}}[w, w]-K_{b, f_{2}}[w, w] \\
& \quad=\left(b^{-1}\left(f_{1}-f_{2}\right) w, f_{1} w\right)+\left(b^{-1} f_{2} w,\left(f_{1}-f_{2}\right) w\right) \\
& \quad-B\left[\left(\Psi_{1}-\Psi_{2}\right) w, \Psi_{1} w\right]-B\left[\Psi_{2} w,\left(\Psi_{1}-\Psi_{2}\right) w\right] .
\end{aligned}
$$

Using (2.9), we see that

$$
\begin{aligned}
& \left|K_{12}[w, w]\right| \leq 2 \underline{b}^{-1}\left(\left\|f_{1} w\right\|+\left\|f_{2} w\right\|\right)\left\|\left(f_{1}-f_{2}\right) w\right\| \\
& \quad \leq C\left\|f_{0} w\right\|\left\|\left(f_{1}-f_{2}\right) w\right\|, \quad f_{0}:=\left(f_{1}^{*} f_{1}+f_{2}^{*} f_{2}\right)^{1 / 2} .
\end{aligned}
$$

Since the forms $A[w, w]$ and $\|\nabla w\|^{2}$ are metrically equivalent, it suffices to consider the quotient

$$
\frac{K_{12}[w, w]}{\|\nabla w\|^{2}}, \quad w \in H_{0}^{1}\left(\Omega, \mathbb{C}^{3}\right) .
$$

By Proposition 4.3, an estimate for this quotient follows from (5.4). Finally, returning to the form $A$, we obtain

$$
\Delta_{3 / 2}\left(T_{1}-T_{2}\right) \leq C\left\|f_{0}\right\|_{L_{3}}^{3 / 2}\left\|f_{1}-f_{2}\right\|_{L_{3}}^{3 / 2} \leq C^{\prime}\left(\left\|f_{1}\right\|_{L_{3}}^{3}+\left\|f_{2}\right\|_{L_{3}}^{3}\right)^{1 / 2}\left\|f_{1}-f_{2}\right\|_{L_{3}}^{3 / 2} .
$$

The same inequality is valid in the case of periodic boundary conditions (the proof is similar). These estimates and (4.6) imply the following statement.

Proposition 5.1. The functionals $d_{3 / 2}((2.12))^{2 / 5}$ and $d_{3 / 2}((3.8))^{2 / 5}$ are Hölder continuous of order $3 / 5$ with respect to the variation of the matrix $f$ in the $L_{3}$-norm.

\section{§6. Reduction of the general case to the CASE of a Cube}

Let $\Omega$ be an open set, and let $Q$ be a cube in $\mathbb{R}^{3}$ such that $\Omega \subset Q$. Let $b$, $\mu$, and $f$ be measurable matrix-valued functions defined in $Q$ and satisfying (2.3)-(2.5). We denote by $\Psi_{\Omega}, K_{\Omega}$, and $A_{\Omega}$ the operator and the forms constructed in $\S 2$ for the set $\Omega$ and the space $H_{0}^{1}\left(\Omega, \mathbb{C}^{3}\right)$, and by $\tilde{\Psi}_{Q}, \tilde{K}_{Q}$, and $\tilde{A}_{Q}$ the operator and the forms constructed in $\S 3$ for the set $Q$ and the space $\tilde{H}^{1}\left(Q, \mathbb{C}^{3}\right)$. For the asymptotic functionals $d_{3 / 2}$ we shall also indicate the corresponding set in the notation: $d_{3 / 2}((2.12), \Omega)$ or $d_{3 / 2}((3.8), Q)$. If a (vector-valued) function belongs to the space $H_{0}^{1}(\Omega)$, then we denote by the same letter the function of class $H_{0}^{1}(Q) \subset \tilde{H}^{1}(Q)$ that extends the initial function by zero. In this section we assume that supp $f \subset \Omega$. We show that, under this condition, $d_{3 / 2}((2.12), \Omega)=$ $d_{3 / 2}((3.8), Q)$.

Consider the auxiliary quotient

$$
\tilde{K}_{Q}[w, w] / \tilde{A}_{Q}[w, w], \quad w \in H_{0}^{1}\left(\Omega, \mathbb{C}^{3}\right) .
$$

Clearly, $\left.\tilde{A}_{Q}\right|_{H_{0}^{1}(\Omega)}=A_{\Omega}$ and $\left.\tilde{B}_{Q}\right|_{H_{0}^{1}(\Omega)}=B_{\Omega}$.

Proposition 6.1. The asymptotic coefficients of the spectrum for the quotients (2.12) and (6.1) coincide: $d_{3 / 2}((2.12), \Omega)=d_{3 / 2}((6.1))$.

Proof. We consider the difference

$$
\frac{\tilde{K}_{Q}[w, w]-K_{\Omega}[w, w]}{A_{\Omega}[w, w]}, \quad w \in H_{0}^{1}\left(\Omega, \mathbb{C}^{3}\right),
$$

of the corresponding quotients and show that

$$
\Delta_{3 / 2}((6.2))=0 .
$$


Setting $\Psi w=\varphi$ and $\tilde{\Psi} w=\tilde{\varphi}$, we transform the numerator of (6.2):

$$
\tilde{K}_{Q}[w, w]-K_{\Omega}[w, w]=B[\varphi, \varphi]-\tilde{B}[\tilde{\varphi}, \tilde{\varphi}]=(f w, \nabla(\varphi-\tilde{\varphi}))=\operatorname{Re}(f w, \nabla(\varphi-\tilde{\varphi})) .
$$

The set $\Omega$ is open, supp $f$ is closed by definition, and supp $f \subset \Omega$. Hence, there is a real-valued function $\zeta \in C_{0}^{\infty}(\Omega)$ such that $\left.\zeta\right|_{\operatorname{supp} f}=1$. We have

$$
(f w, \nabla \varphi)=(f w, \zeta \nabla \varphi)=(f w, \nabla(\zeta \varphi)-(\nabla \zeta) \varphi)=(b \nabla \tilde{\varphi}, \nabla(\zeta \varphi))-(f w,(\nabla \zeta) \varphi) .
$$

By analogy,

$$
(f w, \nabla \tilde{\varphi})=(f w, \nabla(\zeta \tilde{\varphi})-(\nabla \zeta) \tilde{\varphi})=(b \nabla \varphi, \nabla(\zeta \tilde{\varphi}))-(f w,(\nabla \zeta) \tilde{\varphi}) .
$$

Since $\operatorname{Re}(b \nabla \tilde{\varphi}, \zeta \nabla \varphi)=\operatorname{Re}(b \nabla \varphi, \zeta \nabla \tilde{\varphi})$, we obtain

$$
\begin{aligned}
& \tilde{K}_{Q}[w, w]-K_{\Omega}[w, w]=\operatorname{Re}(f w, \nabla(\varphi-\tilde{\varphi})) \\
& \quad=\operatorname{Re}(f w-b \nabla \varphi,(\nabla \zeta) \tilde{\varphi})-\operatorname{Re}(f w-b \nabla \tilde{\varphi},(\nabla \zeta) \varphi) .
\end{aligned}
$$

Therefore,

$$
\left|\tilde{K}_{Q}[w, w]-K_{\Omega}[w, w]\right| \leq C\|f w\|(\|\Psi w\|+\|\tilde{\Psi} w\|) .
$$

Consider the quotients

$$
\begin{aligned}
& \|\Psi w\|^{2} /\|w\|_{H^{1}}^{2}, \quad w \in H_{0}^{1}\left(\Omega, \mathbb{C}^{3}\right), \\
& \|\tilde{\Psi} w\|^{2} /\|w\|_{H^{1}}^{2}, \quad w \in H_{0}^{1}\left(\Omega, \mathbb{C}^{3}\right) .
\end{aligned}
$$

We check the relations

$$
\Delta_{3 / 2}((6.5))=0, \quad \Delta_{3 / 2}((6.6))=0 .
$$

For definiteness, consider the quotient (6.6). If we admit all $w \in \tilde{H}^{1}(Q)$ as test functions in (6.6), then $\Delta_{3 / 2}$ becomes greater. Therefore, $\Delta_{3 / 2}((6.6)) \leq \Delta_{3}(Y \tilde{\Psi})$, where $Y$ is the operator of embedding $\tilde{H}^{1}(Q)$ in $L_{2}(Q)$. Since the operator $Y$ is compact, $(5.5)$ and Proposition 4.1 imply (6.7). Now, using Proposition 4.3, inequality (4.5), and the equivalence of the forms $\|w\|_{H^{1}}^{2}$ and $A_{\Omega}[w, w]$, we see that (6.4) implies (6.3). It remains to refer to Proposition 4.2.

The inequality

$$
n(\lambda,(6.1)) \leq n(\lambda,(3.8), Q)+3
$$

follows from the minimaximal principle. Therefore,

$$
d_{3 / 2}((6.1)) \leq d_{3 / 2}((3.8), Q) .
$$

Taking Proposition 6.1 into account, we obtain the following corollary.

Corollary 6.2. We have

$$
d_{3 / 2}((2.12), \Omega) \leq d_{3 / 2}((3.8), Q) .
$$

Now we prove the reverse inequality.

Proposition 6.3. We have

$$
B\left[\Psi_{\Omega} v, \Psi_{\Omega} v\right] \leq \tilde{B}\left[\tilde{\Psi}_{Q} v, \tilde{\Psi}_{Q} v\right], \quad v \in H_{0}^{1}\left(\Omega, \mathbb{C}^{3}\right) .
$$

Proof. Let $v \in H_{0}^{1}\left(\Omega, \mathbb{C}^{3}\right)$. By definition,

$$
(f v, \nabla \zeta)=\left(b \nabla\left(\tilde{\Psi}_{Q} v\right), \nabla \zeta\right), \quad \zeta \in \tilde{H}^{1}(Q, \mathbb{C}) .
$$

Therefore,

$$
B\left[\Psi_{\Omega} v, \Psi_{\Omega} v\right]=\left(f v, \nabla\left(\Psi_{\Omega} v\right)\right)=\left(b \nabla\left(\tilde{\Psi}_{Q} v\right), \nabla\left(\Psi_{\Omega} v\right)\right)
$$


whence

$$
B\left[\Psi_{\Omega} v, \Psi_{\Omega} v\right] \leq \tilde{B}\left[\tilde{\Psi}_{Q} v, \tilde{\Psi}_{Q} v\right]^{1 / 2} B\left[\Psi_{\Omega} v, \Psi_{\Omega} v\right]^{1 / 2} \Longrightarrow B\left[\Psi_{\Omega} v, \Psi_{\Omega} v\right] \leq \tilde{B}\left[\tilde{\Psi}_{Q} v, \tilde{\Psi}_{Q} v\right] .
$$

Theorem 6.4. Let $Q$ be a cube in $\mathbb{R}^{3}$, and let $\Omega \subset Q$. Suppose that $b$, $\mu$, and $f$ are defined in $Q$ and satisfy (2.3)-(2.5). Moreover, let supp $f \subset \Omega$. Then

$$
d_{3 / 2}((2.12), \Omega)=d_{3 / 2}((3.8), Q) .
$$

Proof. There exist functions $\zeta \in C_{0}^{\infty}(\Omega)$ and $\eta \in \tilde{C}^{\infty}(Q)$ such that

$$
\zeta^{2}+\eta^{2}=1,\left.\quad \zeta\right|_{\operatorname{supp} f}=1 .
$$

For every $u \in \tilde{H}^{1}\left(Q, \mathbb{C}^{3}\right)$ we have

$$
\begin{aligned}
\tilde{A}_{Q}[u, u] & =\left(\mu^{-1} \zeta \operatorname{curl} u, \zeta \operatorname{curl} u\right)+\alpha\|\zeta \operatorname{div} u\|^{2}+\left(\mu^{-1} \eta \operatorname{curl} u, \eta \operatorname{curl} u\right)+\alpha\|\eta \operatorname{div} u\|^{2} \\
& =\tilde{A}_{Q}[\zeta u, \zeta u]+\tilde{A}_{Q}[\eta u, \eta u]+\Phi[u, u],
\end{aligned}
$$

where $\Phi$ is a quadratic form involving the first derivatives of $u$ in the first power only. So, by Proposition 4.4, we can consider the functionals $d_{3 / 2}$ for the quotient

$$
\frac{\tilde{K}_{Q}[u, u]}{\tilde{A}_{Q}[\zeta u, \zeta u]+\tilde{A}_{Q}[\eta u, \eta u]}, \quad u \in \tilde{H}^{1}\left(Q, \mathbb{C}^{3}\right), \quad \int_{Q} u d x=0,
$$

in place of $d_{3 / 2}((3.8), Q)$. The identity $\zeta f=f$ implies that $\tilde{\Psi}_{Q} u=\tilde{\Psi}_{Q}(\zeta u)$ and $\tilde{K}_{Q}[u, u]=\tilde{K}_{Q}[\zeta u, \zeta u]$. Therefore, by (6.8), we have

$$
\begin{aligned}
& \frac{\tilde{K}_{Q}[u, u]}{\tilde{A}_{Q}[\zeta u, \zeta u]+\tilde{A}_{Q}[\eta u, \eta u]} \leq \frac{\left(b^{-1} f \zeta u, f \zeta u\right)-\tilde{B}\left[\tilde{\Psi}_{Q}(\zeta u), \tilde{\Psi}_{Q}(\zeta u)\right]}{\tilde{A}_{Q}[\zeta u, \zeta u]} \\
& \quad \leq \frac{\left(b^{-1} f \zeta u, f \zeta u\right)-B\left[\Psi_{\Omega}(\zeta u), \Psi_{\Omega}(\zeta u)\right]}{\tilde{A}_{Q}[\zeta u, \zeta u]}=\frac{K_{\Omega}[\zeta u, \zeta u]}{A_{\Omega}[\zeta u, \zeta u]}, \quad u \in \tilde{H}^{1}\left(Q, \mathbb{C}^{3}\right) .
\end{aligned}
$$

Let $L$ be a subspace in $\tilde{H}^{1}\left(Q, \mathbb{C}^{3}\right)$ with the following properties:

- $\operatorname{dim} L=n(\lambda,(6.9))$,

$$
\frac{\tilde{K}_{Q}[u, u]}{\tilde{A}_{Q}[\zeta u, \zeta u]+\tilde{A}_{Q}[\eta u, \eta u]}>\lambda \text { for all } u \in L \backslash\{0\}, \int_{Q} u d x=0 .
$$

Observe that if $u \in L \backslash\{0\}$, then $\zeta u \neq 0$, because otherwise $\tilde{K}_{Q}[u, u]=0$. Consequently, $\operatorname{dim}(\zeta L)=\operatorname{dim} L$, where $\zeta L=\{\zeta u: u \in L\}$ is a subspace of $H_{0}^{1}\left(\Omega, \mathbb{C}^{3}\right)$. Formula (6.10) yields

$$
K_{\Omega}[v, v]>\lambda A_{\Omega}[v, v], \quad v \in \zeta L \backslash\{0\}
$$

whence

$$
n(\lambda,(6.9)) \leq n(\lambda,(2.12), \Omega) \Longrightarrow d_{3 / 2}((3.8), Q) \leq d_{3 / 2}((2.12), \Omega) .
$$

The reverse inequality was established above.

Remark 6.5. In particular, if $\Omega=Q$, then $d_{3 / 2}((2.12), Q)=d_{3 / 2}((3.8), Q)$.

Below we shall see that Theorem 6.4 and the continuity of the functionals $d_{3 / 2}$ in the coefficient $f$ imply that it suffices to prove Theorem 3.2 in the case of a cube and periodic boundary conditions only. 


\section{$\S 7$. Estimates of Singular NUMBERS}

In order to prove that the functionals $d_{3 / 2}((3.8), Q)$ are continuous with respect to the coefficients $\mu$ and $b$, we need some auxiliary estimates. In this section we denote by $C(\mu)$ various constants depending on the matrix-valued function $\mu$; they may have different values even within one and the same calculation.

Proposition 7.1. Suppose $\mu \in \tilde{C}^{\infty}(Q)$ satisfies (2.4). Then

$$
\|\psi\|_{H^{2}}^{2} \leq 2 \underline{\mu}^{-2}\|\operatorname{div}(\mu \nabla \psi)\|^{2}+C(\mu)\|\psi\|_{H^{1}}^{2}, \quad \psi \in \tilde{H}^{2}(Q) .
$$

Proof. Let $\psi \in \tilde{C}^{\infty}(Q)$. Integrating by parts, we obtain

$$
\int_{Q} \sum_{i j k l} \mu_{i j} \overline{\mu_{k l}} \partial_{i} \partial_{k} \psi \partial_{j} \partial_{l} \bar{\psi} d x \leq \int_{Q}|\operatorname{div}(\mu \nabla \psi)|^{2} d x+C(\mu)\|\psi\|_{H^{1}}\|\psi\|_{H^{2}} .
$$

It is well known that if a matrix $\mu$ is bounded from below by a number $\underline{\mu}$, then

$$
\sum_{i j k l} \mu_{i j} \overline{\mu_{k l}} \Psi_{i k} \overline{\Psi_{j l}} \geq \underline{\mu}^{2} \sum_{i k}\left|\Psi_{i k}\right|^{2}
$$

for any matrix $\Psi$. Therefore,

$$
\|\psi\|_{H^{2}}^{2} \leq \underline{\mu}^{-2} \int_{Q}|\operatorname{div}(\mu \nabla \psi)|^{2} d x+\frac{1}{2}\|\psi\|_{H^{2}}^{2}+C(\mu)\|\psi\|_{H^{1}}^{2},
$$

which implies $(7.1)$ for $\psi \in \tilde{C}^{\infty}(Q)$. It remains to recall that the space $\tilde{C}^{\infty}(Q)$ is dense in $\tilde{H}^{2}(Q)$.

We introduce the space $\tilde{G}=\left\{\nabla \varphi, \varphi \in \tilde{H}^{1}(Q, \mathbb{C})\right\}$ and its orthogonal complement $\tilde{J}$,

$$
L_{2}\left(Q, \mathbb{C}^{3}\right)=\tilde{J} \oplus \tilde{G} .
$$

Note that the projections of a function $u \in \tilde{H}^{1}\left(Q, \mathbb{C}^{3}\right)$ to the subspaces $\tilde{J}$ and $\tilde{G}$ are also of class $\tilde{H}^{1}\left(Q, \mathbb{C}^{3}\right)$.

Proposition 7.2. Suppose $\mu \in \tilde{C}^{\infty}(Q)$ satisfies (2.4). Let $\tilde{A}$ be the form defined by formula (3.6), and let $\mathcal{A}$ be the corresponding operator in $L_{2}\left(Q, \mathbb{C}^{3}\right)$, restricted to the subspace $\left\{u \in L_{2}\left(Q, \mathbb{C}^{3}\right): \int_{Q} u d x=0\right\}$. Then

$$
\|\operatorname{curl} u\|_{H^{1}}^{2} \leq C_{1}\|\mathcal{A} u\|^{2}+C(\mu)\|\operatorname{curl} u\|^{2}, \quad u \in \operatorname{Dom} \mathcal{A} \cap \tilde{J},
$$

and $\operatorname{curl} u \in \tilde{H}^{1}\left(Q, \mathbb{C}^{3}\right)$. The constant $C_{1}$ depends only on the numbers $\underline{\mu}$ and $\bar{\mu}$.

Proof. Let $u \in \operatorname{Dom} \mathcal{A} \cap \tilde{J}$. Then $\operatorname{div} u=0$ and

$$
\left(\mu^{-1} \operatorname{curl} u, \operatorname{curl} w\right)=(\mathcal{A} u, w), \quad w \in \tilde{H}^{1}\left(Q, \mathbb{C}^{3}\right) .
$$

We write the function $\mu^{-1} \operatorname{curl} u$ in accordance with (7.2),

$$
\mu^{-1} \operatorname{curl} u=v+\nabla \psi, \quad v \in \tilde{J}, \quad \psi \in \tilde{H}^{1}(Q, \mathbb{C}), \quad \int_{Q} \psi d x=0 .
$$

Since $\operatorname{curl} v=\mathcal{A} u$ and $\operatorname{div} v=0$, we have

$$
\|\nabla v\|^{2}=\|\mathcal{A} u\|^{2} .
$$

Next,

$$
(\mu \nabla \psi, \nabla \omega)=-(\mu v, \nabla \omega), \quad \omega \in \tilde{H}^{1}(Q, \mathbb{C}) .
$$

Therefore, $\psi \in \tilde{H}^{2}(Q, \mathbb{C}), \operatorname{div}(\mu \nabla \psi)=-\operatorname{div}(\mu v)$ and, by Proposition 7.1,

$$
\|\psi\|_{H^{2}}^{2} \leq 2 \underline{\mu}^{-2}\|\operatorname{div}(\mu v)\|^{2}+C(\mu)\|\psi\|_{H^{1}}^{2} \leq 4 \bar{\mu}^{2} \underline{\mu}^{-2}\|v\|_{H^{1}}^{2}+C(\mu)\left(\|v\|^{2}+\|\psi\|_{H^{1}}^{2}\right) .
$$


Taking (7.5) and the identity $\|v\|^{2}+\|\nabla \psi\|^{2}=\| \mu^{-1}$ curl $u \|^{2}$ into account, we obtain

$$
\|\psi\|_{H^{2}}^{2} \leq 4 \bar{\mu}^{2} \underline{\mu}^{-2}\|\mathcal{A} u\|^{2}+C(\mu)\|\operatorname{curl} u\|^{2} .
$$

Formulas (7.4), (7.5), and (7.6) imply that

$$
\begin{aligned}
& \|\operatorname{curl} u\|_{H^{1}}^{2}=\|\mu(v+\nabla \psi)\|_{H^{1}}^{2} \leq C\left(\|v\|_{H^{1}}^{2}+\|\psi\|_{H^{2}}^{2}\right)+C(\mu)\|v+\nabla \psi\|^{2} \\
& \quad \leq C_{1}\|\mathcal{A} u\|^{2}+C(\mu)\|\operatorname{curl} u\|^{2} .
\end{aligned}
$$

Remark 7.3. Clearly, inequality (7.3) is true for all $u \in \operatorname{Dom} \mathcal{A} \cap \tilde{G}$ (the left-hand side vanishes) and, thus, for all $u \in \operatorname{Dom} \mathcal{A}$.

Remark 7.4. It can be shown that under the assumptions of Proposition 7.2 we have $\operatorname{Dom} \mathcal{A}=\tilde{H}^{2}\left(Q, \mathbb{C}^{3}\right)$ and

$$
\|u\|_{H^{2}} \leq C\|\mathcal{A} u\|+C(\mu)\|u\|_{H^{1}}, \quad u \in \tilde{H}^{2}\left(Q, \mathbb{C}^{3}\right) .
$$

We shall not use this fact.

Corollary 7.5. Let $\mu$ and $\mathcal{A}$ be the same as in Proposition 7.2 , and let $\rho \in L_{3}(Q)$ be a matrix-valued function. Then for the quotient

$$
\frac{\|\rho \operatorname{curl} u\|^{2}}{\|\mathcal{A} u\|^{2}}, \quad u \in \operatorname{Dom} \mathcal{A} \cap \tilde{J}, \quad \int_{Q} u d x=0,
$$

we have $\Delta_{3 / 2}((7.7)) \leq C_{2}\|\rho\|_{L_{3}}^{3}$, and the constant $C_{2}$ depends only on the numbers $\underline{\mu}$ and $\bar{\mu}$.

Proof. Consider the quotient

$$
\frac{\|\rho v\|^{2}}{\|v\|_{H^{1}}^{2}}, \quad v=\operatorname{curl} u, \quad u \in \operatorname{Dom} \mathcal{A} \cap \tilde{J}, \quad \int_{Q} u d x=0 .
$$

Propositions 7.2 and 4.4 imply that $\Delta_{3 / 2}((7.7)) \leq C \Delta_{3 / 2}((7.8))$. If the domain of a variational quotient becomes larger, then its consecutive maxima do not become smaller. Therefore, it suffices to consider the quotient (7.8) on the functions $v \in \tilde{H}^{1}\left(Q, \mathbb{C}^{3}\right)$. It remains to refer to $(5.4)$.

Remark 7.6. On the set $\operatorname{Dom} \mathcal{A} \cap \tilde{G}$, the quotient (7.7) is identically equal to zero.

Now, we consider the case of two operators $\mathcal{A}$ and $\hat{\mathcal{A}}$.

Proposition 7.7. Let $\mu$ and $\hat{\mu}$ be matrix-valued functions satisfying (2.4), and let $\hat{\mu} \in \tilde{C}^{\infty}(Q)$. The corresponding operators restricted to the subspace $\left\{u \in L_{2}\left(Q, \mathbb{C}^{3}\right)\right.$ : $\left.\int_{Q} u d x=0\right\}$ will be denoted by $\mathcal{A}$ and $\hat{\mathcal{A}}$. Then

$$
\Delta_{3 / 2}\left(\mathcal{A}^{-1}-\hat{\mathcal{A}}^{-1}\right) \leq C_{3}\|\mu-\hat{\mu}\|_{L_{3}}^{3 / 2},
$$

and the constant $C_{3}$ depends only on the numbers $\underline{\mu}$ and $\bar{\mu}$ and on the size of the cube $Q$.

Proof. The decomposition (7.2) reduces the operators $\mathcal{A}$ and $\hat{\mathcal{A}}$, and the parts of these operators in the subspace $\tilde{G}$ coincide. Therefore, it suffices to consider the difference of the inverse operators on the subspace $\tilde{J}$ only. Let

$$
g, h \in \tilde{J}, \quad \int_{Q} g d x=\int_{Q} h d x=0 .
$$

We put

$$
u=\hat{\mathcal{A}}^{-1} g, \quad v=\mathcal{A}^{-1} h \in \tilde{H}^{1}\left(Q, \mathbb{C}^{3}\right) \cap \tilde{J} .
$$


Then

$$
\begin{aligned}
& \left(\hat{\mathcal{A}}^{-1} g, h\right)-\left(g, \mathcal{A}^{-1} h\right)=(u, \mathcal{A} v)-(\hat{\mathcal{A}} u, v) \\
& \quad=\left(\left(\mu^{-1}-\hat{\mu}^{-1}\right) \operatorname{curl} u, \operatorname{curl} v\right)=\left(\left(\mu^{-1}-\hat{\mu}^{-1}\right) \operatorname{curl} \hat{\mathcal{A}}^{-1} g, \operatorname{curl} \mathcal{A}^{-1} h\right) .
\end{aligned}
$$

So,

$$
\left.\hat{\mathcal{A}}^{-1}\right|_{\tilde{J}}-\left.\mathcal{A}^{-1}\right|_{\tilde{J}}=\left.\left(\left.\operatorname{curl} \mathcal{A}^{-1}\right|_{\tilde{J}}\right)^{*}\left(\mu^{-1}-\hat{\mu}^{-1}\right) \operatorname{curl} \hat{\mathcal{A}}^{-1}\right|_{\tilde{J}} .
$$

Furthermore, the operator curl $\left.\mathcal{A}^{-1 / 2}\right|_{\tilde{J}}$ is bounded in $\tilde{J}$, whence, by (4.7),

$$
\Delta_{3}\left(\left.\operatorname{curl} \mathcal{A}^{-1}\right|_{\tilde{J}}\right) \leq C \Delta_{3}\left(\left.\mathcal{A}^{-1 / 2}\right|_{\tilde{J}}\right) \leq C^{\prime}
$$

By Corollary 7.5,

$$
\Delta_{3}\left(\left.\left(\mu^{-1}-\hat{\mu}^{-1}\right) \operatorname{curl} \hat{\mathcal{A}}^{-1}\right|_{\tilde{J}}\right) \leq C_{2}\left\|\mu^{-1}-\hat{\mu}^{-1}\right\|_{L_{3}}^{3} \leq C^{\prime \prime}\|\mu-\hat{\mu}\|_{L_{3}}^{3} .
$$

Now (7.9) follows from (7.10), (4.8), and (4.4).

Theorem 7.8. Let $\mu_{1}$ and $\mu_{2}$ be matrix-valued functions satisfying (2.4), and let $\mathcal{A}_{1}$ and $\mathcal{A}_{2}$ be the corresponding operators. Then

$$
\Delta_{3}\left(\mathcal{A}_{1}^{-1 / 2}-\mathcal{A}_{2}^{-1 / 2}\right) \leq C_{4}\left\|\mu_{1}-\mu_{2}\right\|_{L_{3}}^{3 / 2},
$$

where the constant $C_{4}$ depends only on the numbers $\underline{\mu}$ and $\bar{\mu}$ and on the size of the cube $Q$.

Proof. We construct a matrix-valued function $\hat{\mu} \in \tilde{C}^{\infty}(Q)$ satisfying (2.4) and such that

$$
\left\|\mu_{1}-\hat{\mu}\right\|_{L_{3}}+\left\|\hat{\mu}-\mu_{2}\right\|_{L_{3}} \leq 2\left\|\mu_{1}-\mu_{2}\right\|_{L_{3}}
$$

(this can be done by mollifying the periodic extension of the function $\mu_{1}(x)$ ). Then

$$
\begin{aligned}
& \Delta_{3 / 2}\left(\mathcal{A}_{1}^{-1}-\mathcal{A}_{2}^{-1}\right)^{2 / 5} \leq \Delta_{3 / 2}\left(\mathcal{A}_{1}^{-1}-\hat{\mathcal{A}}^{-1}\right)^{2 / 5}+\Delta_{3 / 2}\left(\hat{\mathcal{A}}^{-1}-\mathcal{A}_{2}^{-1}\right)^{2 / 5} \\
& \quad \leq C_{3}^{2 / 5}\left(\left\|\mu_{1}-\hat{\mu}\right\|_{L_{3}}^{3 / 5}+\left\|\hat{\mu}-\mu_{2}\right\|_{L_{3}}^{3 / 5}\right) \leq 2 C_{3}^{2 / 5}\left\|\mu_{1}-\mu_{2}\right\|_{L_{3}}^{3 / 5}
\end{aligned}
$$

(we have applied Proposition 7.7 and formula (4.5)). Thus,

$$
\Delta_{3 / 2}\left(\mathcal{A}_{1}^{-1}-\mathcal{A}_{2}^{-1}\right) \leq 2^{5 / 2} C_{3}\left\|\mu_{1}-\mu_{2}\right\|_{L_{3}}^{3 / 2} .
$$

It remains to refer to Proposition 4.5.

The following theorem can be established in a similar way (in fact, it is simpler).

Theorem 7.9. Let $b_{1}$ and $b_{2}$ be matrix-valued functions satisfying (2.3), and let $\tilde{B}_{1}$ and $\tilde{B}_{2}$ be the quadratic forms defined by formula $(3.5)$ with $b=b_{j}, j=1,2$. Let $\mathcal{B}_{1}$ and $\mathcal{B}_{2}$ be the corresponding operators restricted to the subspace

$$
\left\{\varphi \in L_{2}(Q, \mathbb{C}): \int_{Q} \varphi d x=0\right\} .
$$

Then

$$
\Delta_{3 / 2}\left(\mathcal{B}_{1}^{-1}-\mathcal{B}_{2}^{-1}\right) \leq C_{5}\left\|b_{1}-b_{2}\right\|_{L_{3}}^{3 / 2},
$$

where the constant $C_{5}$ depends only on $\underline{b}$ and $\bar{b}$ and on the size of the cube $Q$.

An estimate of type (7.12) was established in [5, Chapter 5] for domains with smooth boundary. In essence, the proof of Theorems 7.8 and 7.9 given above is similar to the proof in [5]. These estimates can be extracted also from the more general considerations of [4]. 


\section{$\S 8$. Continuity of the functionals $d_{3 / 2}((3.8))$ WITH RESPECT TO THE COEFFICIENTS $\mu$ AND $b$}

In this section, the constants in estimates depend on the (fixed) matrix $f$. Proposition 5.1 allows us to study only the case where

$$
f \in \tilde{C}^{\infty}(Q) .
$$

As in the preceding section, we denote by $\mathcal{B}$ and $\mathcal{A}$ the selfadjoint positive operators in $L_{2}(Q)$ corresponding to the forms (3.5) and (3.6) and restricted to the subspaces orthogonal to the constants. We also introduce the operators

$$
\begin{aligned}
\mathcal{G} v & =f^{*} b^{-1} f v, & v & \in L_{2}\left(Q, \mathbb{C}^{3}\right), \\
\mathcal{F} u & =\mathcal{B} \tilde{\Psi} u=-\operatorname{div}(f u), & & u \in \tilde{H}^{1}\left(Q, \mathbb{C}^{3}\right), \\
\mathcal{F}^{*} \psi & =f^{*} \nabla \psi, & & \in \tilde{H}^{1}(Q, \mathbb{C}) .
\end{aligned}
$$

Note that, under the assumption (8.1), the operators $\mathcal{G}, \mathcal{F} \mathcal{A}^{-1 / 2}$ and $\mathcal{F}^{*} \mathcal{B}^{-1} \mathcal{F}$ are continuous in $L_{2}\left(Q, \mathbb{C}^{3}\right)$. It is easily seen that the spectrum of the quotient (3.8) coincides with the spectrum of the operator

$$
\mathcal{A}^{-1 / 2}\left(\mathcal{G}-\mathcal{F}^{*} \mathcal{B}^{-1} \mathcal{F}\right) \mathcal{A}^{-1 / 2}
$$

which is selfadjoint and compact on $L_{2}\left(Q, \mathbb{C}^{3}\right)$.

Suppose that the Hermitian matrix-valued functions $b_{j}$ and $\mu_{j}, j=1,2$, have the same bounds as in (2.3) and (2.4). Consider the difference of two operators of type (8.2) (with $b_{1}, \mu_{1}$ and $b_{2}, \mu_{2}$ ). We have

$$
\begin{aligned}
& \mathcal{A}_{1}^{-1 / 2}\left(\mathcal{G}_{1}-\mathcal{F}^{*} \mathcal{B}_{1}^{-1} \mathcal{F}\right) \mathcal{A}_{1}^{-1 / 2}-\mathcal{A}_{2}^{-1 / 2}\left(\mathcal{G}_{2}-\mathcal{F}^{*} \mathcal{B}_{2}^{-1} \mathcal{F}\right) \mathcal{A}_{2}^{-1 / 2} \\
& =\left(\mathcal{A}_{1}^{-1 / 2}-\mathcal{A}_{2}^{-1 / 2}\right)\left(\mathcal{G}_{1}-\mathcal{F}^{*} \mathcal{B}_{1}^{-1} \mathcal{F}\right) \mathcal{A}_{1}^{-1 / 2}+\mathcal{A}_{2}^{-1 / 2}\left(\mathcal{G}_{1}-\mathcal{F}^{*} \mathcal{B}_{1}^{-1} \mathcal{F}\right)\left(\mathcal{A}_{1}^{-1 / 2}-\mathcal{A}_{2}^{-1 / 2}\right) \\
& \quad+\mathcal{A}_{2}^{-1 / 2}\left(\mathcal{G}_{1}-\mathcal{G}_{2}\right) \mathcal{A}_{2}^{-1 / 2}+\mathcal{A}_{2}^{-1 / 2} \mathcal{F}^{*}\left(\mathcal{B}_{2}^{-1}-\mathcal{B}_{1}^{-1}\right) \mathcal{F} \mathcal{A}_{2}^{-1 / 2} \\
& =: T_{1}+T_{2}+T_{3}+T_{4} .
\end{aligned}
$$

By (4.5), (4.6), it suffices to estimate $\Delta_{3 / 2}$ for each of the four terms on the right hand side. By (4.7), (4.8), and (7.11), we have

$$
\Delta_{3 / 2}\left(T_{1}\right) \leq 2 C_{4}^{1 / 2}\left\|\mu_{1}-\mu_{2}\right\|_{L_{3}}^{3 / 4}\left\|\mathcal{G}_{1}-\mathcal{F}^{*} \mathcal{B}_{1}^{-1} \mathcal{F}\right\|^{3 / 2} \Delta_{3}\left(\mathcal{A}_{1}^{-1 / 2}\right)^{1 / 2} \leq C\left\|\mu_{1}-\mu_{2}\right\|_{L_{3}}^{3 / 4} .
$$

The quantity $\Delta_{3 / 2}\left(T_{2}\right)$ is estimated in the same way. The quantity $\Delta_{3 / 2}\left(T_{3}\right)$ is estimated with the help of (5.4):

$$
\Delta_{3 / 2}\left(T_{3}\right) \leq C\left\|b_{1}-b_{2}\right\|_{L_{3 / 2}}^{3 / 2}
$$

Finally, (7.12) implies that

$$
\Delta_{3 / 2}\left(T_{4}\right) \leq C\left\|b_{1}-b_{2}\right\|_{L_{3}}^{3 / 2} .
$$

Combining Proposition 5.1 with the estimates obtained above, we arrive at the following statement.

Proposition 8.1. The functionals $d_{3 / 2}((3.8), Q)$ are continuous in the $L_{3}(Q)$-norm with respect to simultaneous variation of the coefficients $f, \mu$, and $b$. 


\section{§9. Model PRoblem}

In this section we study the case of constant coefficients. Let $Q$ be a cube with edge $l$. Suppose that the matrices $\mu, b$, and $f$ do not depend on $x$. We consider the spectrum of the quotient (3.8). Let $\lambda$ be an eigenvalue of the operator whose quadratic form coincides with $\tilde{K}[w, w]$ in the Hilbert space $\left\{u \in \tilde{H}^{1}\left(Q, \mathbb{C}^{3}\right): \int_{Q} u d x=0\right\}$ with the metric (3.6). If $u$ is the corresponding eigenfunction, then

$$
\left(b^{-1} f u, f w\right)-\tilde{B}[\tilde{\Psi} u, \tilde{\Psi} w]=\lambda \tilde{A}[u, w] \quad \text { for all } w \in \tilde{H}^{1}\left(Q, \mathbb{C}^{3}\right) \text { such that } \int_{Q} w d x=0 .
$$

Set $\varphi=\tilde{\Psi} u$. By definition,

$$
\tilde{B}[\tilde{\Psi} u, \tilde{\Psi} w]=(\nabla \varphi, f w), \quad w \in \tilde{H}^{1}\left(Q, \mathbb{C}^{3}\right) .
$$

Hence, the spectrum of the quotient (3.8) coincides with the spectrum of the following system of partial differential equations:

$$
\left\{\begin{aligned}
f^{*} b^{-1} f u(x)-f^{*} \nabla \varphi(x) & =\lambda\left(\operatorname{curl}\left(\mu^{-1} \operatorname{curl} u(x)\right)-\alpha \nabla \operatorname{div} u(x)\right), \\
\operatorname{div}(b \nabla \varphi(x)) & =\operatorname{div}(f u(x)), \\
u \in \tilde{H}^{1}\left(Q, \mathbb{C}^{3}\right), \quad \int_{Q} u d x & =0, \quad \varphi \in \tilde{H}^{1}(Q, \mathbb{C}), \quad \int_{Q} \varphi d x=0 .
\end{aligned}\right.
$$

There is no loss of generality in assuming that $Q=(0, l)^{3}$. The pairs of the form

$$
u(x)=h e^{2 \pi i m x / l}, \varphi(x)=\psi e^{2 \pi i m x / l}, \quad m \in \mathbb{Z}^{3} \backslash\{0\}, h \in \mathbb{C}^{3}, \psi \in \mathbb{C},
$$

where $m, h$, and $\psi$ satisfy the relations

$$
\left\{\begin{aligned}
f^{*} b^{-1} f h-2 \pi i l^{-1} \psi f^{*} m & =4 \pi^{2} \lambda l^{-2}\left(r(m) \mu^{-1} r(m) h+\alpha\langle h, m\rangle m\right), \\
-4 \pi^{2} l^{-2} \psi\langle b m, m\rangle & =2 \pi i l^{-1}\langle f h, m\rangle,
\end{aligned}\right.
$$

form a full system of eigenfunctions of problem (9.1). Finding $\psi$ from the second equation, we reduce problem (9.2) to the problem

$$
f^{*} b^{-1} f h-\frac{\langle f h, m\rangle}{\langle b m, m\rangle} f^{*} m=\frac{4 \pi^{2} \lambda}{l^{2}}\left(r(m) \mu^{-1} r(m) h+\alpha\langle h, m\rangle m\right), \quad h \in \mathbb{C}^{3} .
$$

Thus, the spectrum of the variational quotient (3.8) with constant coefficients in a cube with edge $l$ coincides with the union over $m \in \mathbb{Z}^{3} \backslash\{0\}$ of the spectra of the problems (9.3). Therefore,

$$
n(\lambda,(3.8))=\sum_{j=1}^{3} \#\left\{m \in \mathbb{Z}^{3} \backslash\{0\}: \lambda_{j}(m)>4 \pi^{2} l^{-2} \lambda\right\},
$$

where the $\lambda_{j}(\xi)$ are the eigenvalues of (3.1).

Proposition 9.1. Let a continuous function $\lambda$ in $\mathbb{R}^{3} \backslash\{0\}$ be homogeneous of order -2 , i.e., $\lambda(t \xi)=t^{-2} \lambda(\xi)$. Then

$$
\#\left\{m \in \mathbb{Z}^{3} \backslash\{0\}: \lambda(m)>\beta\right\} \sim \operatorname{meas}\left\{\xi \in \mathbb{R}^{3}: \lambda(\xi)>\beta\right\}, \quad \beta \rightarrow+0 .
$$

Proof. It is clear that

$$
\operatorname{meas}\left\{\xi \in \mathbb{R}^{3}: \lambda(\xi)>\beta\right\}=\beta^{-3 / 2} \operatorname{meas}\left\{\xi \in \mathbb{R}^{3}: \lambda(\xi)>1\right\} .
$$

On the other hand,

$$
\begin{aligned}
& \beta^{3 / 2} \#\left\{m \in \mathbb{Z}^{3} \backslash\{0\}: \lambda(m)>\beta\right\}=\beta^{3 / 2} \#\left\{\hat{m} \in(\sqrt{\beta} \mathbb{Z})^{3} \backslash\{0\}: \lambda(\hat{m})>1\right\} \\
& \underset{\beta \rightarrow+0}{\longrightarrow} \operatorname{meas}\left\{\xi \in \mathbb{R}^{3}: \lambda(\xi)>1\right\} .
\end{aligned}
$$


Since the functions $\lambda_{j}(\xi)$ are homogeneous of order -2 , formula (9.4) shows that

$$
n(\lambda,(3.8)) \sim(2 \pi)^{-3} l^{3} \sum_{j=1}^{3} \operatorname{meas}\left\{\xi \in \mathbb{R}^{3}: \lambda_{j}(\xi)>\lambda\right\}, \quad \lambda \rightarrow+0 .
$$

We have proved the following statement.

Proposition 9.2. Let $Q$ be a cube with edge $l$ in $\mathbb{R}^{3}$. Suppose that the coefficients $\mu, b$, and $f$ do not depend on $x$. Then

$$
n(\lambda,(3.8)) \sim\left(\frac{l}{2 \pi}\right)^{3} \int_{\mathbb{R}^{3}} n(\lambda ; \xi) d \xi, \quad \lambda \rightarrow+0,
$$

where $n(\lambda ; \xi)$ is the counting function of the spectrum for problem (3.1) with constant $\mu$, $b$, and $f$.

\section{§10. Proof of Theorem 3.2}

First, we establish the additivity of the functionals $d_{3 / 2}$.

Proposition 10.1. Let $Q, Q_{j}, j=1, \ldots, M$, be open cubes in $\mathbb{R}^{3}$, and let

$$
\bar{Q}=\bigcup_{j=1}^{M} \bar{Q}_{j}, \quad Q_{i} \cap Q_{j}=\varnothing \text { for } i \neq j .
$$

Then

$$
d_{3 / 2}((3.8), Q)=\sum_{j=1}^{M} d_{3 / 2}\left((3.8), Q_{j}\right) .
$$

Proof. Set $\Xi=\bigcup_{j=1}^{M} Q_{j}$. Proposition 5.1 allows us to assume without loss of generality that supp $f \subset \Xi$. For the Dirichlet problem, we have

$$
n(\lambda,(2.12), \Xi)=\sum_{j=1}^{M} n\left(\lambda,(2.12), Q_{j}\right) \Longrightarrow d_{3 / 2}((2.12), \Xi)=\sum_{j=1}^{M} d_{3 / 2}\left((2.12), Q_{j}\right) .
$$

It remains to note that Theorem 6.4 implies that

$$
d_{3 / 2}((3.8), Q)=d_{3 / 2}((2.12), \Xi), \quad d_{3 / 2}\left((3.8), Q_{j}\right)=d_{3 / 2}\left((2.12), Q_{j}\right) .
$$

Proof of Theorem 3.2. By Proposition 5.1, the functionals $d_{3 / 2}((2.12), \Omega)$ are continuous in the $L_{3}(\Omega)$-norm with respect to the coefficient $f$. Therefore, without loss of generality, we may assume that supp $f \subset \Omega$. Let $Q$ be a cube containing $\Omega$. We extend the coefficients $f, \mu$, and $b$ to $Q \backslash \Omega$ : $f$ is extended by zero, and $\mu$ and $b$ are extended so as to preserve conditions (2.4) and (2.3). By Theorem 6.4, it suffices to prove the relation (10.1)

$$
n(\lambda,(3.8), Q) \sim(2 \pi)^{-3} \int_{\Omega} \int_{\mathbb{R}^{3}} n(\lambda ; x, \xi) d x d \xi=(2 \pi)^{-3} \int_{Q} \int_{\mathbb{R}^{3}} n(\lambda ; x, \xi) d x d \xi, \quad \lambda \rightarrow+0
$$

(we have used (3.2) at the last step). By Proposition 8.1 and Remark 3.3, the two sides of (10.1) are continuous in the $L_{3}(Q)$-norm with respect to all coefficients. Thus, it suffices to prove (10.1) for piecewise constant coefficients. Suppose that the cube $Q$ is represented as a union of disjoint cubes $Q_{j}$,

$$
\bar{Q}=\bigcup_{j=1}^{M} \bar{Q}_{j}, \quad Q_{i} \cap Q_{j}=\varnothing \text { for } i \neq j,
$$


and that the matrices $f(x), \mu(x)$, and $b(x)$ are constant on each $Q_{j}$. Propositions 9.2 and 3.1 imply that

$$
\begin{aligned}
& d_{3 / 2}\left((3.8), Q_{j}\right)=\lim _{\lambda \rightarrow+0} \lambda^{3 / 2} n\left(\lambda,(3.8), Q_{j}\right) \\
& =\frac{\operatorname{meas} Q_{j}}{24 \pi^{3}} \int_{|\theta|=1} \sum_{k=1}^{3} \lambda_{k}(x, \theta)^{3 / 2} d S(\theta), \quad x \in Q_{j} .
\end{aligned}
$$

Now we use Proposition 10.1:

$$
\begin{aligned}
& \Delta_{3 / 2}((3.8), Q)=\delta_{3 / 2}((3.8), Q)=\sum_{j=1}^{M} \lim _{\lambda \rightarrow+0} \lambda^{3 / 2} n\left(\lambda,(3.8), Q_{j}\right) \\
& \quad=\frac{1}{24 \pi^{3}} \int_{Q} \int_{|\theta|=1} \sum_{k=1}^{3} \lambda_{k}(x, \theta)^{3 / 2} d x d S(\theta) .
\end{aligned}
$$

Thus, the limit $\lim _{\lambda \rightarrow+0} \lambda^{3 / 2} n(\lambda,(3.8), Q)$ exists, and (3.3) and (10.2) imply (10.1).

\section{REFERENCES}

[1] A. B. Alekseev, Spectral asymptotics of elliptic boundary value problems with solvable constraints, Candidate Diss., Leningrad. Gos. Univ., Leningrad, 1977. (Russian)

[2] A. B. Alekseev and M. Sh. Birman, Asymptotic behavior of the spectrum of elliptic boundary value problems with solvable constraints, Dokl. Akad. Nauk SSSR 230 (1976), no. 3, 505-507; English transl., Soviet Math. Dokl. 17 (1976), 1319-1322 (1977). MR0420027 (54:8044)

[3] M. Sh. Birman, L. S. Koplienko, and M. Z. Solomyak, Estimates of the spectrum of a difference of fractional powers of selfadjoint operators, Izv. Vyssh. Uchebn. Zaved. Mat. 1975, no. 3 (154), 3-10; English transl., Soviet Math. (Iz. VUZ) 19 (1975), no. 3, 1-6 (1976). MR0385597 (52:6458)

[4] M. Sh. Birman and M. Z. Solomyak, Spectral asymptotics of nonsmooth elliptic operators. I, II, Trudy Moskov. Mat. Obshch. 27 (1972), 3-52; ibid. 28 (1973), 3-34; English transl., Trans. Moscow Math. Soc. 27 (1972), 1-52 (1975); ibid. 28 (1973), 1-32 (1975). MR0364898 (51:1152)

[5] _ Quantitative analysis in Sobolev imbedding theorems and applications to spectral theory, Tenth Mathematical School (Summer School, Kaciveli/Nalchik, 1972), Inst. Mat. Akad. Nauk Ukrain. SSR, Kiev, 1974, pp. 5-189; English transl., Amer. Math. Soc. Transl. (2), vol. 114, Amer. Math. Soc., Providence, RI, 1980. MR0482138 (58:2224); MR0562305 (80m:46026)

[6] Weyl asymptotics of the spectrum of the Maxwell operator for domains with a Lipschitz boundary, Vestnik Leningrad. Univ. Mat. Mekh. Astronom. 1987, vyp. 3, 23-28; English transl., Vestnik Leningrad Univ. Math. 20 (1987), no. 3, 15-21. MR0928156 (89h:35253)

[7] _ Spectral theory of selfadjoint operators in Hilbert space, Leningrad. Gos. Univ., Leningrad, 1980; English transl., Math. Appl. (Soviet Ser.), D. Reidel Publishing Co., Dordrecht, 1987. MR0609148 (82k:47001); MR1192782 (93g:47001)

[8] - On the main singularities of the electric component of the electro-magnetic field in regions with screens, Algebra i Analiz 5 (1993), no. 1, 143-159; English transl., St. Petersburg Math. J. 5 (1994), no. 1, 125-139. MR1220492 (94f:35137)

[9] M. Sh. Birman and N. D. Filonov, Weyl asymptotics of the spectrum of the Maxwell operator with non-smooth coefficients in Lipschitz domains, Nonlinear Equations and Spectral Theory, Amer. Math. Soc. Transl. Ser. 2, vol. 220, Amer. Math. Soc., Providence, RI, 2007, pp. 27-44. 
[10] M. Cwikel, Weak type estimates for singular values and the number of bound states of Schrödinger operators, Ann. of Math. (2) 106 (1977), 93-100. MR0473576 (57:13242)

[11] H. Weyl, Über das Spektrum der Hohlraumstrahlung, J. Reine Angew. Math. 141 (1912), $163-181$.

Department of Physics, St. Petersburg State University, Ul'yanovskaya 1, Petrodvorets, St. Petersburg 198504, Russia

Department of Physics, St. Petersburg State University, Ul'yanovskaya 1, Petrodvorets, St. Petersburg 198504, Russia

E-mail address: mbirman@list.ru

Department of Physics, St. Petersburg State University, Ul'yanovskaya 1, Petrodvorets, St. Petersburg 198504, Russia

E-mail address: laugre@mail.ru

Received 4/AUG/2006

Translated by N. D. FILONOV 